\title{
Correction to: Alterations of brain local functional connectivity in amnestic mild cognitive impairment
}

Dan Zheng ${ }^{1 \dagger}$, Wei Xia ${ }^{2 \dagger}$, Zhong Quan Yi ${ }^{2}$, Pan Wen Zhao ${ }^{2}$, Jian Guo Zhong ${ }^{3}$, Hai Cun Shi ${ }^{3}$, Hua Liang Li ${ }^{3}$, Zhen Yu Dai ${ }^{4^{*}}$ and Ping Lei Pan ${ }^{2,3^{*}}$

\section{Correction to: Transl Neurodegener https://doi.org/10.1186/s40035-018-0134-8}

In the original publication of this article [1] surname of the first author is wrong. The correct name of the first author should be Dan Zheng. The original publication has been corrected.

\begin{abstract}
Author details
${ }^{1}$ School of Nursing, Jiangsu Vocational College of Medicine, Yancheng, People's Republic of China. ${ }^{2}$ Department of Neurology, Affiliated Yancheng Hospital, School of Medicine, Southeast University, West Xindu Road 2\#, Yancheng, Jiangsu Province 224001, People's Republic of China. ${ }^{3}$ Department of Central Laboratory, Affiliated Yancheng Hospital, School of Medicine, Southeast University, West Xindu Road 2\#, Yancheng, Jiangsu Province 224001, People's Republic of China. ${ }^{4}$ Department of Radiology, Affiliated Yancheng Hospital, School of Medicine, Southeast University, West Xindu Road 2\#, Yancheng, Jiangsu Province 224001, People's Republic of China.
\end{abstract}

Received: 10 December 2018 Accepted: 10 December 2018 Published online: 24 December 2018

\section{Reference}

1. Zheng D, et al. Alterations of brain local functional connectivity in amnestic mild cognitive impairment. Transl Neurodegener. 2018;7:26 https://doi.org/ 10.1186/s40035-018-0134-8.

\footnotetext{
* Correspondence: daiycsy@sina.com; panpinglei@163.com

${ }^{\dagger}$ Dan Zheng and Wei Xia contributed equally to this work.

${ }^{4}$ Department of Radiology, Affiliated Yancheng Hospital, School of Medicine, Southeast University, West Xindu Road 2\#, Yancheng, Jiangsu Province 224001, People's Republic of China

${ }^{2}$ Department of Neurology, Affiliated Yancheng Hospital, School of Medicine, Southeast University, West Xindu Road 2\#, Yancheng, Jiangsu Province 224001, People's Republic of China

Full list of author information is available at the end of the article
}

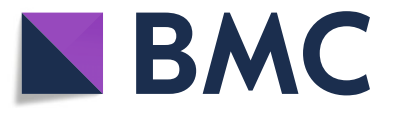

(c) The Author(s). 2018 Open Access This article is distributed under the terms of the Creative Commons Attribution 4.0 International License (http://creativecommons.org/licenses/by/4.0/), which permits unrestricted use, distribution, and reproduction in any medium, provided you give appropriate credit to the original author(s) and the source, provide a link to the Creative Commons license, and indicate if changes were made. The Creative Commons Public Domain Dedication waiver (http://creativecommons.org/publicdomain/zero/1.0/) applies to the data made available in this article, unless otherwise stated. 